BMC

Genomics

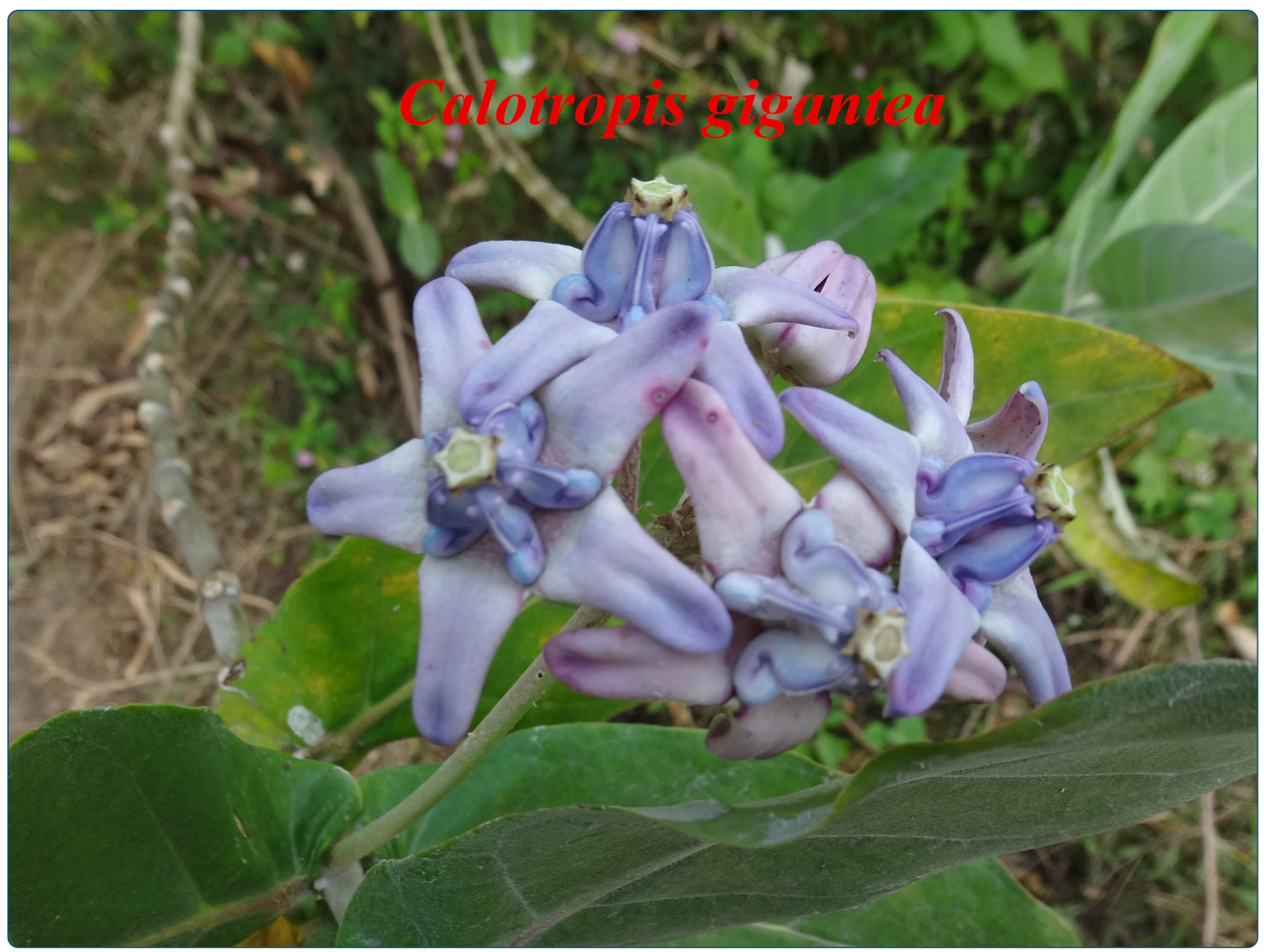

De novo sequencing and assembly analysis of transcriptome in the Sodom apple (Calotropis gigantea)

Muriira et al. 


\title{
De novo sequencing and assembly analysis of transcriptome in the Sodom apple (Calotropis gigantea)
}

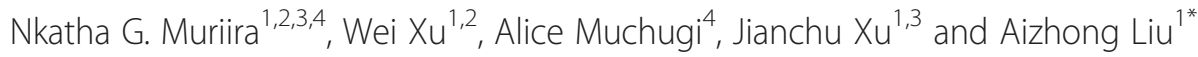

\begin{abstract}
Background: The Sodom apple (Calotropis gigantea), a member of the Asclepiadaceae family, is a large evergreen shrub native to continental Asia and northern Africa. As an important medicinal shrub and a fiber resource plant, there is an urgent need for developing molecular markers to facilitate breeding and genetic improvement of varieties.

Results: In this study, using the Illumina high throughput sequencing technique we obtained about 45 million paired end sequencing reads, De novo assembled and generated a total of 133,634 transcripts with a mean of 1837.47 bp in length. Based on protein homology searches against available databases, a total of 21,851 unigenes were functionally annotated. In particular, many transcripts that encode for putative proteins involved in fiber and secondary metabolite biosynthesis were identified and analyzed. Key fiber genes identified were validated experimentally through Real-Time PCR technique. Various transcription factors involved in regulating plant response to abiotic stress were also identified. In addition, based on the unigene sequences assembled, 11,623 microsatellites loci were detected, which provide very useful resources for developing microsatellite molecular markers.
\end{abstract}

Conclusion: This study is the first report on transcriptome information in the Calotropis species and provides rich gene transcript resources for conducting further studies on understanding the molecular basis of fiber and secondary metabolite biosynthesis, serving the genetic improvement and resource utilization in Calotropis plants.

Keywords: Sodom apple, Calotropis gigantea, High throughput sequencing, Transcriptome, Fiber biosynthesis, Secondary metabolite biosynthesis

\section{Background}

The Sodom apple, also called Crown Flower or Giant Milkweed, belongs to the Asclepiadaceae family and is a large evergreen shrub native to tropics and subtropics of Asia and Africa. Since its seed coat produces fine, silky, durable, and high-quality fiber which is used broadly in industrial applications, Sodom apple is known as Crystal Cotton [1, 2]. The genus Calotropis has two species: Calotropis gigantea (native to continental Asia and South-East Asia) and C. procera (native to northern Africa and southern Asia) [3, 4]. Although they are morphologically similar in that their leaves are opposite, broad, sub-sessile and

\footnotetext{
* Correspondence: liuaizhong@mail.kib.ac.cn

${ }^{1}$ Key Laboratory for Economic Plants and Biotechnology, Kunming Institute of Botany, Chinese Academy of Sciences, Lanhei Road 132, Heilongtan, Kunming 650201, Yunnan, China

Full list of author information is available at the end of the article
}

ovoid follicles, sub-globose, or oblong-lanceolate, and inflated mesocarp [1] with fiber embedded beneath the seeds, taxonomically, C. gigantea is distinguished from $C$. procera by its reflexed corolla with five petals which are white to pale lilac blue [1].

With cotton prices soaring in recent years, the textile industry worldwide is now facing severe challenges [2]. Exploring new fiber resources to enrich the fiber supply has drawn great attention in the industry. Due to the high-quality fiber produced with the seed coat and its characteristics of being fast-growing, drought hardy, and having wide adaptation to soil conditions, developing the cultivation of Sodom apple to provide fine and highquality fiber materials has created great interest in many countries [5]. Also, Sodom apple has been used as a source of medicine because its milk-like latex contains various active-compounds such osmotin and lupeol, and 
other plant parts contain cardiac glycosides, flavonoids, phenolic compounds, and terpenoides [6,7], which are responsible for the various it's pharmacological properties [8]. Due to its potential economic importance, the Sodom apple has been introduced to the Pacific Islands, Australia, as well as to Central and South America [4]. In recent years, efforts on planting Sodom apples for fiber or medicine resources have been employed in many regions [1, 8]. However, Sodom apple is still an undomesticated plant with uncertain economic returns. To facilitate breeding and improvement of varieties, there is an urgent need for investigating the molecular basis of traits concerned with fiber biosynthesis and developing molecular markers in Sodom apple.

Transcriptomic data provide a great opportunity for discovering novel genes and collecting a number of ESTs (Expressed Sequence Tags), which facilitates the development of molecular markers, in particular for non-model organisms without a reference genome $[9,10]$. In this study, we de novo assembled and characterized the transcriptome of Sodom apple. In particular, we detected various novel transcripts involved in fiber biosynthesis. To our knowledge, this study is the first report on characterizing the complete transcriptome data in the genus Calotropis, serving as a valuable resource for novel gene discovery, molecular marker development and genetic improvement in practice.

\section{Results}

\section{Illumina paired-end sequencing data and De novo assembly}

In total, our high throughput sequencing generated approximately 45 million paired end reads. After stringent quality checking and data cleaning, approximately $11 \mathrm{~Gb}$ reads were obtained with $97.46 \%$ Q20 bases with a GC content of $43.42 \%$. An assembler, trinity developed specifically for use with next-generation short-read sequences [10], was employed for De novo assembly. The raw sequence data generated was deposited at the NCBI (National Center for Biotechnology Information) Short Read Archive under accession number SRX1020451. We assembled a total of 133,634 transcripts with a mean of 1837.47. The transcripts were further clustered into 50,742 unigenes with a mean length of $858.83 \mathrm{bp}$ and N50 value of $1733 \mathrm{bp}$. Among the unigenes, 6,987 (13.77 \%) unigenes were greater than $1 \mathrm{~kb}$ and 17,887 (35.25 \%) were between 200-300 bp. Since the shorter sequences may lack a characterized protein domain or may be too short to show sequence matches, resulting in false-negative results, the contigs which were less than 200 bp in length were excluded in our homology searches (see Table 1). The length distributions of the unigenes are shown in Fig. 1. Sequence annotations of all unigenes were predicted with HMMER parameter E-value
Table 1 Summary of sequencing data and De novo assembling

\begin{tabular}{lll}
\hline Length Range & Transcripts & Unigenes \\
\hline $200-300$ & $20,106(15.05 \%)$ & $17,887(35.25 \%)$ \\
$300-500$ & $15,275(11.43 \%)$ & $11,701(23.06 \%)$ \\
$500-1000$ & $17,139(12.83 \%)$ & $8,237(16.23 \%)$ \\
$1000-2000$ & $30,573(22.88 \%)$ & $6,987(13.77 \%)$ \\
$2000+$ & $50,541(37.82 \%)$ & $5,930(11.69 \%)$ \\
Total Number & 133,634 & 50,742 \\
N50 Length & 2,909 & 1,733 \\
Mean Length & 1837.47 & 858.83 \\
\hline
\end{tabular}

of not more than $10^{-10}$ or BLAST (Basic Local Alignment Search Tool) with an E-value threshold of $10^{-5}$ in the NCBI database of non-redundant protein $(\mathrm{Nr})$, along with the Swiss-Prot protein database, the Kyoto Encyclopedia of Genes and Genomes (KEGG) database, the Clusters of Orthologous Groups (COG) database, Eukaryotic Orthologous Groups of proteins (KOG), protein families (Pfam), and the Gene ontology (GO) database. Finally, after sequence annotation, 21,851 (43.06 \%) unigenes were predicted and 28,891 (56.94\%) unigenes are still unknown.

\section{Functional annotation}

All 50,742 unigenes were annotated by aligning with the deposited ones in diverse protein databases mentioned above. As a result, 21,851 (43.06 \%) were successfully annotated. Among these annotated unigenes, 21,243 $(41.86 \%)$ had significant marches in the $\mathrm{Nr}$ database, 16,339 (32.2 \%) had significant marches to Pfam database, 16,150 (31.83 \%) had significant marches to GO database, while 15,117 (29.79\%) had marches to the Swiss-Prot database (see Fig. 2). In total, 50,742 unigenes obtained were BLAST for homology searches, resulting in 21,851 (43.06 \%) unigenes showing similarity to known protein databases. The E-value distribution of the top hits in the $\mathrm{Nr}$ database revealed that $67.67 \%$ of the mapped sequences showed significant homology (less than $1.0 \mathrm{E}^{-50}$ ) while $27.63 \%$ had an E-value between $1.0 \mathrm{E}^{-50}$ and $1.0 \mathrm{E}^{-10}$ (Fig. 3a). Score distribution showed that $41.69 \%$ of the unigenes had scores $>500$ and $35.25 \%<300$ (Fig. 3b). For unigene sequences in the NR annotations, as shown in Fig. 3c, BLAST search analysis further revealed that a total $6,165(28.21 \%)$ had the most similar sequences to proteins from Solanum lycopersicum, followed by Vitis vinifera, 3,909 (17.88 \%), Theobroma cacao, 1,786 (8.14 \%).

\section{Functional classification}

In order to classify the functions of predicted unigenes, GO analysis (offering a dynamic-updated controlled vocabulary and a strictly-defined concept to comprehensively describe 


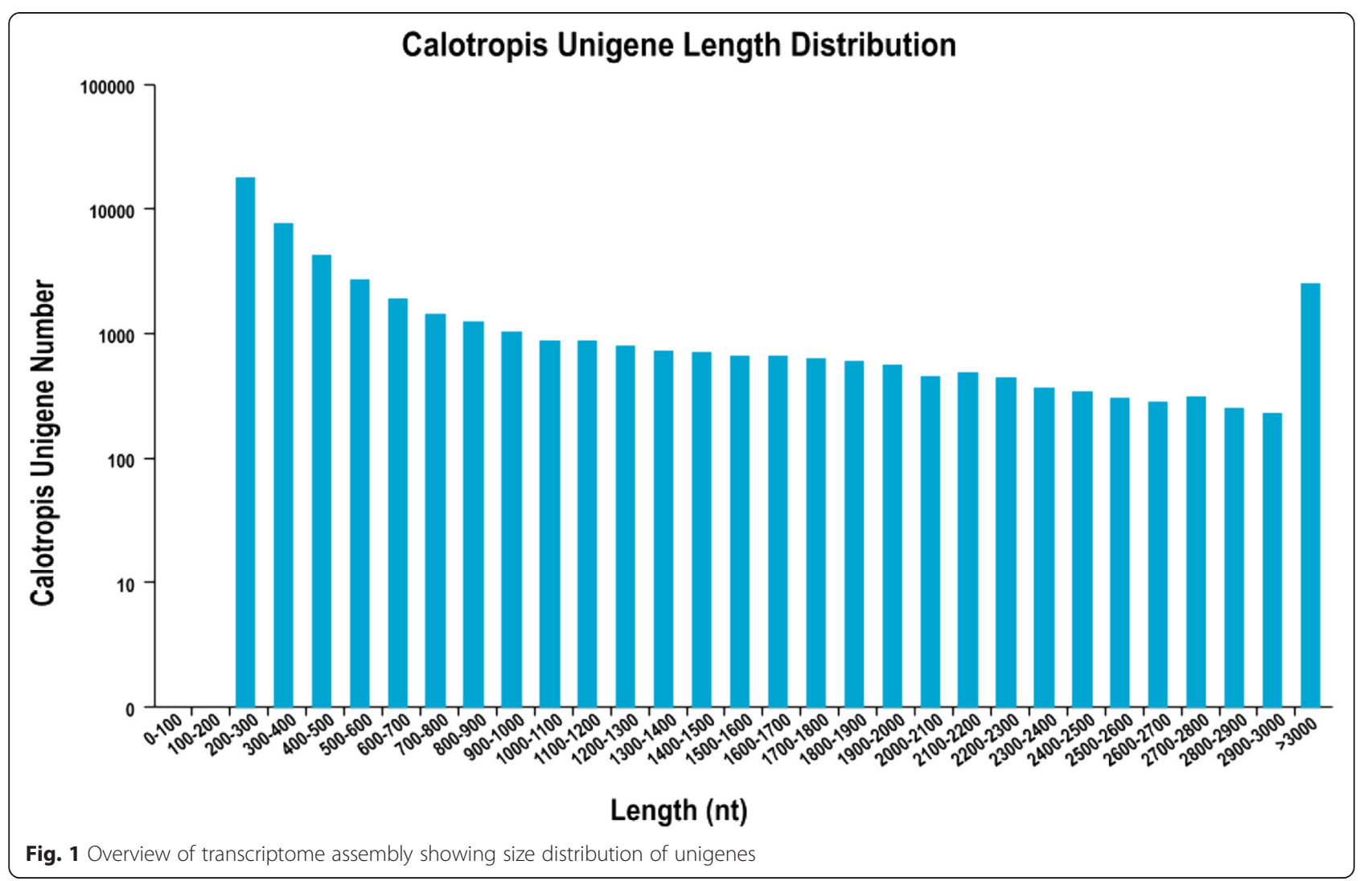

the properties of genes and their products in any organism) was applied. Based on the GO annotation, a total of 16,150 unigenes could be assigned to one or more terms, which were categorized into 53 functional groups under three main divisions (Fig. 4). In the biological function group, the marched unique sequences 48,285 (49.71\%) were clustered into 20 classes. In this category, proteins highly encoded were those involved in metabolic process $(10,826$ unigenes, $20 \%)$, followed by cellular process $(9,838$ unigenes, $18.40 \%)$, single-organism process $(8,831$ unigenes, $16.51 \%)$, response to stimulus (4,750 unigenes, $8.88 \%)$,



Fig. 2 The unigene number annotated in the public database searches and biological regulation (3,979 unigenes, $7.44 \%)$. In the molecular function group, these unique sequences (24,732; $25.46 \%)$ were divided into 17 groups. The catalytic activity (8,024 unigenes, $42.45 \%)$ and binding (7,592 unigenes, $40.17 \%$ ) were the predominant groups followed by, transporter activity (1,200 unigenes, $6.34 \%)$ and structural molecule activity (669 unigenes, 3.34\%). Cellular components were grouped into 16 classes, within which the assignments were mostly enriched in the cell part $(24,111 ; 24.82 \%)$ and cell (11,054 unigenes, $24.59 \%)$ followed by (8,846 unigenes, $19.68 \%)$, membrane $(4,868$ unigene, $10.83 \%)$, and organelle part (3,179 unigenes, $7.07 \%$ ) (Fig. 4).

To further predict the gene function and evaluate the completeness of the transcriptome library, all the assembled unigenes were searched against the clusters of orthologous groups (COG) database. Overall, 8,406 marched unique sequences were clustered into 25 functional categories (Fig. 5). The proteins in the COG categories were assumed to have the same ancestor protein or to be paralogs or orthologs. The largest category was translation, ribosomal structure, and biogenesis with (974; $8.39 \%$ ), and transcripts associated with transcription repair (967; $8.33 \%)$ were most common, followed by replication, recombination, and repair $(962 ; 8.28 \%)$ and signal transduction mechanisms (840; 7.23 \%) (Fig. 5). In addition, 324 unigenes (3.30\%) of COG annotated unigenes were 


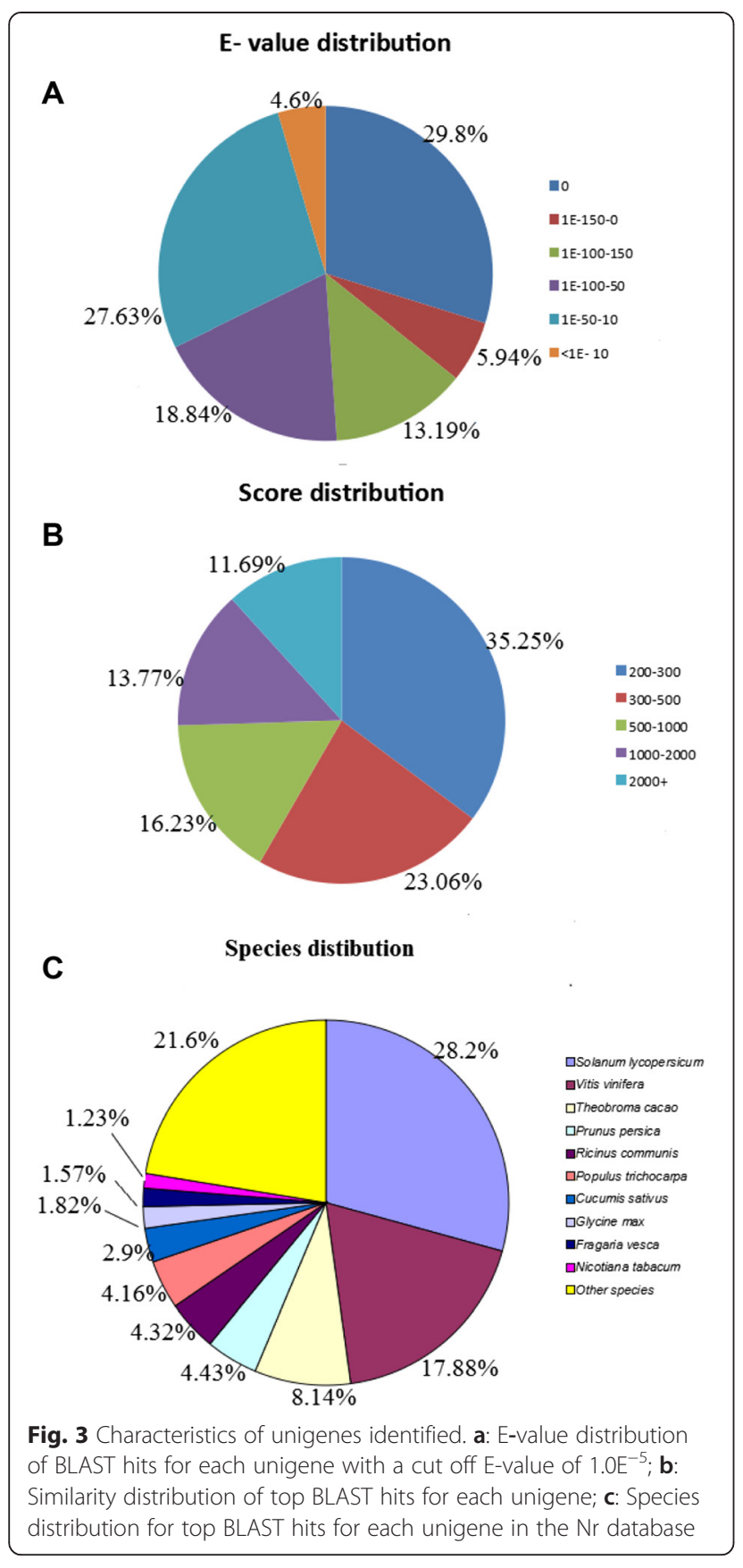

assigned to secondary metabolites biosynthesis, transport and catabolism reflected the large amount of secondary metabolites present in the Sodom apple. However, cell motility and nuclear structure were assigned nine and one unigenes, respectively, whereas no unigene was assigned to extracellular structures (Fig. 5). It was found that categories with no concrete assignment, such as function unknown $(2.78 \%)$ and general function prediction only (18.33\%), accounted for a large fraction of transcripts (Fig. 5).

\section{KEGG classification}

The KEGG pathway database records the networks of molecular interactions in the cells and their variants specific to particular organisms. All the unigenes were analyzed against the KEGG pathway database. In total, 5,391 (10.62 \%) sequences were identified in the KEGG database and were assigned to 116 KEGG pathways (see Additional file 1), covering five major KEGG categories, including metabolism (54.76 \%), genetic information processing (33.30\%), environmental information processing (3.66 \%), cellular processes $(5.15 \%)$ and organismal systems (3.08 \%) (Fig. 6a). Among them, 1,687 unigenes were assigned to the metabolic pathways category. Biosynthesis of secondary metabolites (735) and ribosome (462) were also highly represented (Fig. 6b). Within the metabolic pathway, carbohydrate metabolism was the most highly represented with starch and sugar metabolism, a pathway involved in cellulose biosynthesis the main component of Sodom apple fiber [2], being assigned 120 unigenes (Fig. 6c). In the biosynthesis of secondary metabolites category, 253 unigenes were classified into 13 subcategories, the most prominent being phenylpropanoid biosynthesis (66 unigenes), terpenoid backbone biosynthesis (39 unigenes), tropane, piperidine and pyridine alkaloid biosynthesis (24 unigenes), carotenoid biosynthesis (24 unigenes), flavonoid biosynthesis (22 unigenes), and zeatin biosynthesis (22 unigenes). In addition, other metabolites included isoquinoline alkaloid biosynthesis (16 unigenes), diterpenoid biosynthesis (12 unigenes), limonene and pinene degradation (10 unigenes), stilbenoid, diarylheptanoid and gingerol biosynthesis (10 unigenes), brassinosteroid biosynthesis (5 unigenes), caffeine metabolism (3 unigenes), and flavone and flavonol biosynthesis (2 unigenes) (Fig. 6c). Several genes encoding biosynthesis of secondary metabolites were identified (Table 2). These unigenes involved in metabolism pathways could provide a critical clue for identifying novel genes involved in biosynthesis of highquality fiber and unique chemical compounds in Calotropis plants.

\section{Identification of genes involved in fiber biosynthesis in \\ Sodom apple}

As reported, cellulose, lignin, and pectin are major components of fiber and their content in the fiber could significantly influence the yield and quality of fiber [2]. The genes for cellulose and pectin biosynthesis were identified in the starch-sugar pathway where a total of 120 genes were annotated in the KEGG pathway, whereas genes involved in lignin biosynthesis were found in the phenylpropanoid pathway where 66 unigenes were annotated to the pathway (Fig. 7).

While focusing on identification of novel genes involved in fiber biosynthesis in Sodom apple, we detected 



Fig. 5 Histogram presentation of COG function classification. A total of 8,406 unigenes showing significant homology to the COG database within NCBI (E-value $\leq 1.0$ e - 5) were classified into 25 categories 


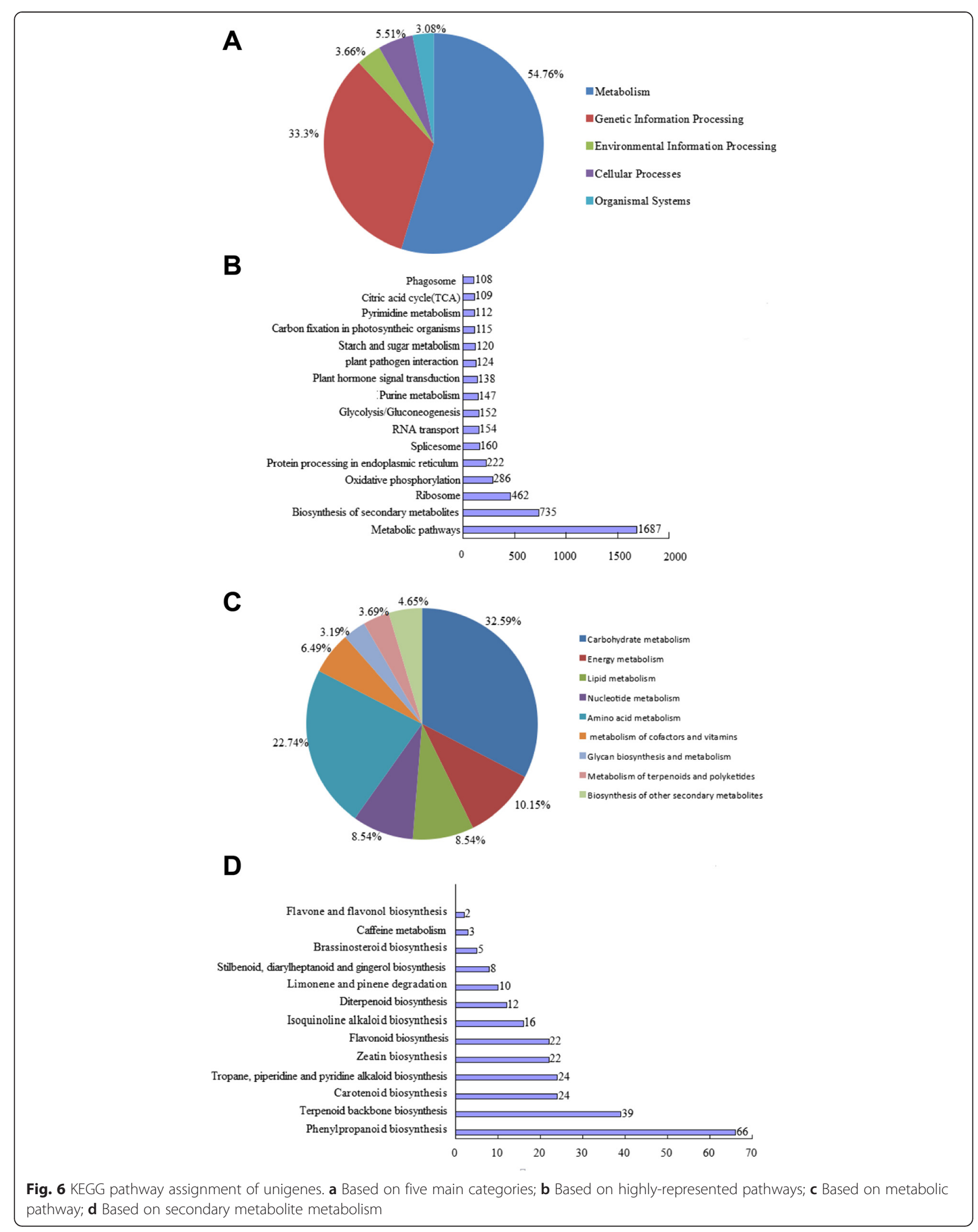


Table 2 Transcripts involved in secondary metabolite biosynthesis

\begin{tabular}{lll}
\hline Unigene id & KO id & Annotated Gene name \\
\hline c22156 & K00626 & Acetyl-CoA C-acetyltransferase \\
c22353 & K00869 & Mevalonate kinase, putative \\
c21763 & K01641 & Hydroxymethylglutaryl-CoA synthase, putative \\
c7727 & K01662 & Deoxyxylulose-5-phosphate synthase, putative \\
c14940 & K01662 & Deoxyxylulose-5-phosphate synthase, putative \\
c23487 & K016621 & Deoxyxylulose-5-phosphate synthase, putative \\
c17748 & K00231 & Amine oxidase, putative \\
c12793 & K01592 & Amino acid decarboxylase, putative \\
c29332 & K09755 & Flavonoid 3-hydroxylase, putative \\
c48278 & K09755 & Flavonoid 3-hydroxylase, putative \\
c17911 & K09755 & Flavonoid 3-hydroxylase, putative \\
c24158 & K09755 & Flavonoid 3-hydroxylase, putative \\
c28297 & K00475 & Flavanone 3-hydroxylase, putative \\
c12264 & K05278 & Flavonol synthase \\
c26268 & K10775 & Phenylalanine ammonia-lyase, putative \\
C21787 & K00487 & Cinnamate 4-hydroxylase, putative \\
c23419 & K01904 & 4CL \\
c26322 & K01904 & 4CL1 \\
\hline
\end{tabular}

33 genes involved in cellulose, lignin, and pectin biosynthesis. In particular, one unigene encoding PGM (phosphoglucomutase) enzyme, one unigene for UGP2 (glucose 1 phosphate uridyldyltransferase), five unigenes encoding enzyme cellulose synthase (CesA) (UDP-forming synthase) were identified. Genes involved in Pectin biosynthesis, one unigene encoding GAE (UDP-D-glucuronate-4-epimerase), one unigene encoding GAUT (alpha-1,4- galacturonosyltransferase), and seven unigenes encoding pectin modifying enzymes pectin esterases were also found (Additional file 2). For lignin biosynthesis, transcripts encoding various enzymes were identified. They included, one unigene encoding phenylalanine ammonia-lyase, one unigene encoding cinnamate 4-hydroxylase, three unigenes encoding 4Hydroxycinnamoyl CoA ligase, two unigenes encoding cinnamoyl $\mathrm{CoA}$ reductase and 11 unigenes encoding peroxidase (Additional file 2; Fig. 7). The identification of genes involved in metabolism pathways gives a potential basis for gene discovery and cloning from Sodom apple.

\section{Identification of transcription factors involved in abiotic stress response}

As previously reported, the Sodom apple is drought and salt tolerant [5]. Identification of potential transcription factors involved in regulating physiological responses to abiotic stress might add additional understanding in the potential molecular mechanisms of drought and salt tolerance in Sodom apple. We identified various transcription factor families which might be involved in regulatory response to abiotic stress, including $B T F 3$ (two unigenes), $M Y B$ (214 unigenes), AP2/Ethylene-responsive transcription factor $1 \mathrm{~B}(89)$ and bZIP (Basic leucine zipper protein) (47) unigenes transcription factors (see Additional file 3). In particular, many identified transcripts with putative transcription factors have not been assigned to any known transcription factors.

\section{Validation of unigenes and gene expression profiling using Real-Time PCR}

In order to experimentally validate the reliability of the unigenes obtained from the assembled transcriptome and gene expression profile, four unigenes involved in fiber biosynthesis, such as the unigenes c8180, c20855, c21961 and c24331 encoding a key cellulose synthase CesA, were tested using Real-Time PCR. As shown in Fig. 8, the expression profiles of the four unigenes tested among different tissues exhibited that all the transcripts were expressed in the tested tissues. Apart from unigene c24331 in which leaf showed highest expression, the highest expression for three genes was in developing seed coats, in consistent with that the seed coat tissue is the most active in fiber biosynthesis.

\section{Simple sequence repeats (SSR) loci discovery}

To develop molecular markers of marker-assisted selection for genetic breeding and improvement, the 50,742 unigenes generated were used to mine potential microsatellites by using MISA software. A total of 12,917 unique sequences were examined, resulting in an identification of 11,623 putative microsatellites (including 5265 mononucleotides), covering 9,606 perfect SSR loci, and 943 loci in a compound form (among which 2,886 sequences contained more than one SSR loci). The most abundant repeat motif was dinucleotide, 3,701 (58.2 \%), followed by trinucleotides, 2,418 (38.03\%), tetranucleotide, 208 (3.27 \%), pentanucleotide, 17 (0.27 \%) and hexanucleotide, $14(0.22 \%)$. SSRs with six tandem repeats (26.95 \%) were most common, followed by five ( $24.3 \%)$, seven (17.96\%), eight (11.07\%), nine (10.71\%) and $>10$ (2.12\%) (Table 3). AT/TA motifs constituted more than half $(62.7 \%)$ of the total number of dinucleotides. Further, 5,692 SSR primer pairs were successfully designed using software Primer 3. The details of the frequency of SSR motif and genic-SSR primers sequences (including designing parameters) are summarized in Additional file 4. These SSR loci would provide a great way to develop polymorphic SSR markers in Sodom apple.

\section{Discussion}

\section{Characterization of the Sodom apple transcriptome}

Transcriptome sequencing has become an important tool because of its low cost and high throughput $[11,12]$. In the recent past, many De novo transcriptome data on 

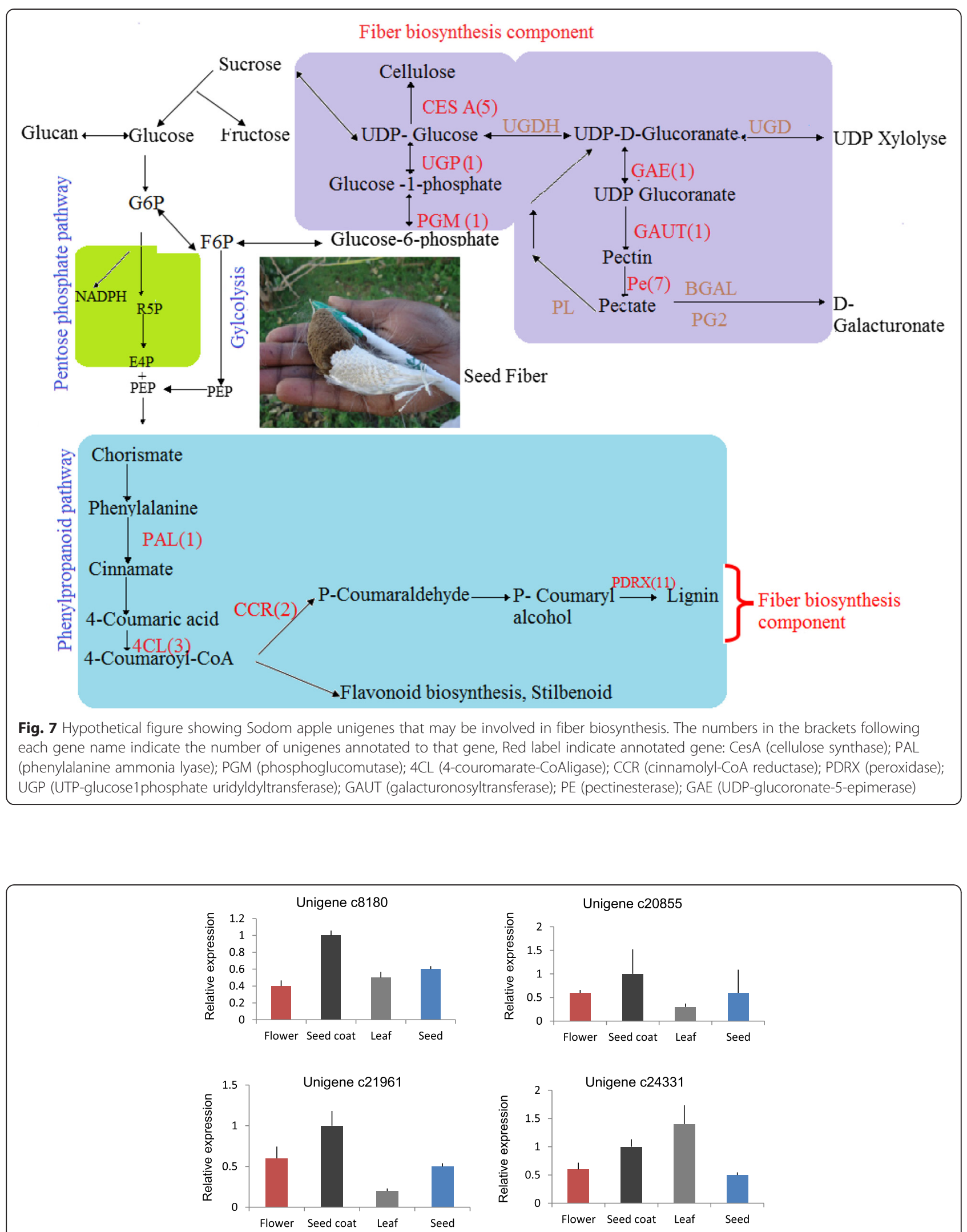

Fig. 8 Real-Time PCR analysis of four unigenes associated with the fiber biosynthesis. Error bars were calculated based on three replicates. Actin gene (c26600) was used as an internal control for normalization 
Table 3 Distribution and frequency of EST-SSRs identified in Sodom apple

\begin{tabular}{|c|c|c|c|c|c|c|c|c|c|}
\hline \multirow[t]{2}{*}{ Motif types } & \multicolumn{9}{|c|}{ Repeat numbers } \\
\hline & 5 & 6 & 7 & 8 & 9 & 10 & $>10$ & total & $\%$ \\
\hline Dinucleotide & 0 & 1015 & 763 & 676 & 678 & 436 & 133 & 3701 & 58.2 \\
\hline Trinucleotide & 1346 & 664 & 378 & 26 & 3 & 0 & 1 & 2418 & 38.03 \\
\hline Tetra & 180 & 26 & 1 & 1 & 0 & 0 & 0 & 208 & 3.27 \\
\hline Penta & 14 & 3 & 0 & 0 & 0 & 0 & 0 & 17 & 0.27 \\
\hline Hexa & 5 & 6 & 0 & 1 & 0 & 1 & 1 & 14 & 0.22 \\
\hline Total & 1545 & 1714 & 1142 & 704 & 681 & 437 & 135 & 6358 & \\
\hline$\%$ & 24.3 & 26.95 & 17.96 & 11.072 & 10.71 & 6.87 & 2.12 & & \\
\hline
\end{tabular}

non-model plants including Centella asiatica [13], Ramia [14], Liriodendron chinense [15], Gossypium aridum [16], and Gossypium hirsutum [17] have been generated by using the Illumina high throughput sequencing technology. These transcriptome data provide valuable resources for further studies in these plants.

In this study, the transcriptome sequencing was carried out using the Illumina Genome Analyser system platform HiSeq2500 read-length of $125 \mathrm{bp}$, which is longer than other sequencing platforms, such as Hiseq2000, and much faster. More than 45 million high-quality reads were used to de novo assemble the transcriptome of various tissues. The reads were assembled into 50,742 unigenes with an average length of $858.80 \mathrm{bp}$ and an N50 of $1,733 \mathrm{bp}$, which is used for assembly evaluation and a high number [18], suggest a high quality assembly in this study. The Q20 of $94.23 \%$ with a GC value of $43.42 \%$ also reflects a high quality sequencing run [19]. The protein homology searches revealed that Sodom apple unigenes had highest similarity to Solanum lycopersicum (28.20\%) genes, implying that Sodom apple may phylogenically be closer to Solanum plants than to other eudict plants for which genome annotation has been done, such as Vitis vinifera, Populus trichocarpa and Glycine max. These results indicate that our current high throughput sequencing data and transcriptome assembly are of high quality and these data will provide a solid foundation for further gene discovery, marker development, marker-assisted selection breeding, and genetic improvement studies in Sodom apple.

\section{Transcriptome assembly and gene annotation}

In this study, 21,851 (43.06\%) unigenes out of 50,742 identified were successfully annotated using BLAST searches of the public nr, Pfam, Swiss-Prot, GO, COG, and KEGG databases. This means that more than half of unigenes generated were not annotated according to existing databases. This could be as a result of several reasons such as the absence of genomic information on the family Asclepiadaceae, the unigenes without hits probably belonged to untranslated regions, they could also have been non-coding RNA, short sequences not containing a protein domain, or assembly mistakes [20]. Since genomic and transcriptomic information is currently lacking in the family Asclepiadaceae in databases, these unigenes without hits may be considered putative novel transcribed sequences. Therefore, according to these results, there is a need to generate a large collection of unigenes and further characterize the gene structures and expression patterns in Sodom apple.

The best hit for each unigene queried against the NCBI $\mathrm{Nr}$ database was utilized to assign functional GO annotation in terms of biological process, molecular function, and cellular component groups (Fig. 4). The large number of diverse $\mathrm{GO}$ assignments to unigenes highlights the diversity of genes likely represented in the transcriptome data. The functions of the identified genes cover various molecular functional categories which was the highest GO ontology, and the well-represented categories included ATP binding (GO:0005524) metal ion binding (GO:0046872), DNA binding (GO:0003677) zinc ion binding (GO:0008270), nucleotide binding (GO:0000166), protein binding (GO:0005515), and the structural constituent of ribosome (GO:0003735). The sequences encoded a broad set of transcripts represented within the molecular component category which indicates the need for a large number of transcripts for carrying out various biochemical processes. The results demonstrated that high throughput transcriptome sequencing is an efficient, reliable, and inexpensive tool for transcriptome analysis in Sodom apple. These gene annotations would provide a valuable resource for investigating specific physiological processes, gene structures and functions, and metabolism pathways in Sodom apple.

\section{Potential candidate genes involving fiber biosynthesis}

De novo transcriptome assembly and characterization based on high throughput sequencing technology has enabled the rapid identification of candidate genes involved in various biosynthesis pathways such as Carotenoid genes in Liriodendron Chinese [15], lipid genes in Sacha Inchi [21], Steroidal Sapogen genes in Asparagus racemosus [22], CesA gene in ramie (Boehmeria nivea L. 
Gaud) [14], and lignin biosynthesis genes in Celery [23], among others. As an important fiber plant, dissecting the molecular mechanism of fiber development in Sodom apple is an attractive research area with a focus on novel gene discovery, improving fiber quality, yield, and genetic improvement. Carbohydrate and energy metabolisms play an important role in the fiber development by providing the carbon skeletons for the synthesis of cell wall polysaccharides [24]. UDP-D-glucose is a central metabolite in carbohydrate metabolism and is the common precursor for synthesis of cell wall polysaccharides such as pectin, hemicelluloses, and cellulose. In cellulose UDP-D, glucose is involved in the formation of cellulose synthase (UDP-forming synthase) (CesAs) central catalysts involved in initiation and elongation of plant cell wall cellulose [25], which is a component of Sodom apple fiber.

Cellulose is the main cell wall polysaccharides important in fiber growth and development [26]. CesA genes have been the cellulose synthase most extensively studied in plants such as Ramia, Arabidopsis, rice and cotton [14, 27-29]. In this study, unigenes encoding CesA were identified on the basis of KEGG database searches, of which $61 \%$ of Sodom apple fiber is constituted of cellulose [2]. The Real-Time PCR experiments revealed that the four tested CesA genes were highly expressed in seed coat tissues where fibers are actively biosynthesized, strongly suggesting that these genes are involved in the biosynthesis of fibers in Sodom apple. Further studies focusing on characterizing the function of $\operatorname{Ces} A$ genes would provide clues in understanding the mechanisms of fiber development in Sodom apple.

As Sodom apple fiber does not undergo secondary cell wall deposition, pectin is an important fiber component. In the present study, several transcripts encoding enzymes involved in synthesis of pectin were found, 1-4 galacturonosyltransferase, a key enzyme for pectin biosynthesis [30], catalyzes 1,4 D-galacturonosyl to galacturonic acid, the primary pectic polysaccharide of the plant primary wall [31]. UTP-glucose-1-phosphate uridylyltransferase (UGP2) catalyzes the formation of UDPglucose from glucose-1-phosphate and UTP, involved in the formation of UDP-D-glucoranate while UDP-Dglucuronate-5-epimerase $(G A E)$ converts UDP-a-D-glucuronic acid to UDP-galacturonic a precursor of pectin [32]. Galacturonosyltransferase (GAUT) transfers galacturonic acid from uridine 5'-diphosphogalacturonic acid onto the pectic polysaccharide homogalacturonan [33]. In addition, gene encoding pectin modifying enzymes pectin esterases which might play a major role in the fiber cell wall development were also identified (Additional file 2; Fig. 7) in our current study.

Lignin is another component of Sodom apple fiber consisting of about $21 \%$ of fiber content after cellulose
(66\%) and pectin (3\%) [3]. Lignins are complex racemic aromatic heteropolymers that are derived from monolignols which are products of the phenylpropanoid metabolism [34]. Among the lignin biosynthesis transcripts identified from our transcriptome, $P A L$ is the first enzyme of the phenylpropanoid pathway and catalyzes the deamination of phenylalanine to produce trans-cinnamic acid. Cinnate 4-hydroxylase $(\mathrm{C} 4 \mathrm{H})$, belonging to the CYP73A group of cytochrome P450-dependent monooxygenases protein family, hydroxylates cinnamic acid to generate $p$-coumaric acid, whereas, 4-Couramarate CoA $(4 C L)$ is responsible for the CoA esterification of $p$-coumaric acid, caffeic acid, ferulic acid, 5-hydroxyferulic acid, and sinapic acid. In addition, the Peroxidase enzyme promotes polymerization of monolignols resulting in lignins [34] (Additional file 2; Fig. 7).

According to lignin metabolic pathways five genes required to encode the related enzymes were found in our transcriptome data set. The existence of lignin in Sodom apple has a negative effect on their dye uptake and dye fixation [2] which affects the utilization of this fiber for textile industry. Thus, understanding its biosynthesis is critical in improvement of this fiber. For example, it has been found that down regulating $4 C L$ can decrease lignin content while increasing cellulose components [35].

\section{Secondary metabolites identified}

A wide range of chemical compounds have been isolated from the Sodom apple, including glycosides [36], flavonoids and triterpenoids [37], cardiac glycosides [38], phenolic compounds, and terpenoides [6] which are associated with its medicinal potential. The KEGG pathway analysis of all unigenes identified supported these results by the presence of a large number of transcripts involved in secondary metabolite biosynthesis.

Terpenoids are an important class of secondary metabolites in Sodom apple; two pathways directly related to terpenoid biosynthesis (terpenoid backbone biosynthesis and diterpenoid biosynthesis) were present in our transcriptome. Terpenoids are synthesized through two main pathways, the mevalonate and pyruvate/glyceraldehyde-3-phosphate pathways [39]. In our transcriptome data, several of the transcripts annotated to these two pathways including acetyl-CoA C- acetyltransferase, hydroxymethylglutaryl-CoA synthase, mevalonate kinase, and 1- deoxyxylulose-5-phosphate synthase. The zeatin biosynthesis and carotenoid biosynthesis pathways related to terpenoid biosynthesis were annotated in our library. Alkaloids are other important secondary metabolites that are mainly involved in defense and are responsible for many of the medicinal properties of plants [40]. KEGG pathway analysis of our transcriptome revealed two secondary metabolite pathways directly related to alkaloid biosynthesis, i.e., isoquinoline alkaloid biosynthesis and 
tropane, piperidine, and pyridine alkaloid biosynthesis (Fig. 6c). Since amino acids are the main precursors of all alkaloids, we observed transcripts for several enzymes in alkaloid biosynthetic pathways such as amine oxidase, amino transferase, and amino acid decarboxylase. In addition, flavonoids are also important secondary metabolites that perform a variety of essential functions in higher plants [41]. Among the secondary metabolite pathways in Sodom apple, transcripts annotated to the main enzymes of the flavonoid biosynthesis and flavonol biosynthesis pathways were observed in our transcriptome, including flavonoid-3-hydroxylase, flavanone-3-hydroxylase, flavonol synthase (Table 2). The phenylpropanoid biosynthesis pathway, which was the most highly represented secondary metabolite pathway (Fig. 6c), is also closely related to flavonoid biosynthesis because the metabolite phenylalanine is the only precursor for flavonoid biosynthesis [42]. Several important phenylpropanoid biosynthetic enzymes linking primary metabolism to flavonoid biosynthesis were observed in our transcriptome, including phenylalanine ammonia lyase, cinnamate 4-hydroxylase, and 4- coumarate-CoA ligase. Overall, $3.3 \%$ of the COG annotated unigenes fell into the secondary metabolite biosynthesis, transport, and catabolism category (Fig. 5). Our functional classification of Sodom apple genes indicates the presence of a large number of active secondary metabolite processes, providing strong transcriptomic evidence to support previous biochemical observations.

\section{Identification of transcription factor involved in stress response}

Transcription factors usually play crucial roles in adjusting plant adaptation to adverse environments. Sodom apple is a deep rooted, wild shrub well-acclimatized to salinity and drought [5]. Transcript profiling can be a significant tool for the characterization of stress-responsive genes transcriptional factor. Among transcription factors identified from our data, BTF3 is one of the most important transcription factors due to their role in various biotic and abiotic stress processes and different physiological and developmental mechanisms such as ionic homeostasis in plants [43]. The AP2s and ERFs have been found to be involved in various biotic and abiotic stress responses [44]. In particular, bZIPs have been known to be involved in salt and drought tolerance [45]. Both MYC and MYB TFs have been found to participate in the abscissic acid (ABA)dependent pathway of stress signaling for the upregulation of the abiotic stress responsive genes [46] which could possibly be the case even in the Sodom apple. However, it needs further functional validations on whether these transcription factors identified from Sodom apple have specific function in increasing salt and drought tolerance. The presence of these transcription factors identified in our transcriptome data in this study would guide further gene discovery and functional experiments for their important characterization in further genetic improvement.

\section{SSR discovery}

SSR molecular markers are locus-specific, co-dominant, multi-allelic, highly polymorphic, and transferable among species within genera [47]. EST-SSR markers are very important for studies involving genetic diversity, cultivar identification, evolution, linkage mapping, QTL mapping, comparative genomics, and marker-assisted selection breeding [48]. High throughput transcriptome sequencing provides plenty of SSR loci for molecular marker development. The 11,623 putative microsatellites located on unigenes obtained from Sodom apple provided very helpful resources for designing primers (Additional file 4) which will be used for developing molecular markers and serving various research purposes in Sodom apple.

\section{Conclusion}

The focus of this study was to employ the Illumina high throughput sequencing platform to characterize and assemble the transcriptome of C. gigantea in order to provide a large transcriptome sequence dataset. In this study, we obtained 50,742 unigenes with an average length of $858.8 \mathrm{bp}$. Importantly, we found many transcripts that encode for putative proteins that are involved in fiber and secondary metabolite biosynthesis, and the fiber candidate genes were validated using the Real-Time PCR method. Various transcription factors related to stress were also identified. This study demonstrated that high throughput transcriptome sequencing is an efficient, reliable, and in expensive tool for transcriptome analysis and marker discovery in Sodom apple. To our knowledge, this is the first report on investigating the whole transcriptome data using high throughput sequencing technology in the genus Calotropis. We trust that this dataset will be valuable in improving further research on molecular mechanisms of fiber biosynthesis, stress tolerance, and as a resource for future improvement through marker-assisted breeding and genetic diversity studies in the Sodom apple.

\section{Methods}

\section{Plant materials and RNA extraction}

In order to capture the full transcriptome serving various researches in Sodom apple, different tissues such as shoot, leaf, flower, seed coat and seed were included. Leaf tissues were collected from three developing stages determined by the leaf size; flower tissues included buds, opening and mature flowers; seed coat tissues included the developing seed coats and its young fibers; seed tissues included the developing seeds and the mature seeds. Tissues were collected from three individuals to 
make biological replicates. The collected tissues were immediately frozen in liquid nitrogen, stored in $-80{ }^{\circ} \mathrm{C}$ until RNA isolation. Total RNA was isolated and purified using $\mathrm{RnaEx}^{\mathrm{Tm}}$ (GENEray Shanghai, China) according to the manufacturer's protocol and treated with RNasefree DNASE I (GENEray Shanghai, China) to remove DNA contamination. RNA integrity and quality was evaluated with $1.0 \%$ formaldehyde agarose gel and Nano-drop 2000 spectrophotometer (Thermo scientific). Further, RNA quality and purity was verified using 2100 Agilent Bioanalyzer and Qubit 2.0. Equal quantities of high-quality RNA from each of the four RNA extractions were pooled for cDNA synthesis to ensure we obtained full transcriptome.

\section{CDNA library preparation and transcriptome sequencing} High throughput sequencing was performed at Biomarker Biotechnology Co., Ltd. China, using HiSeq 2500 sequencing platform. After the qualified RNA samples were pooled, magnetic beads with oligo (dT) were used to isolate poly (A) mRNA from total RNA, according to the Illumina manufacturer's instructions. The purified mRNA was then disrupted into short fragments using fragmentation buffer. Using these short fragments as templates, first cDNA strand was synthesized using random hexamer primers. A Second-strand cDNA was then synthesized using buffer, dNTPs, RNaseH, and DNA polymerase I. The synthesized cDNA were purified with AMPure XP beads and resolved with EB buffer for end repair and poly (A) addition. Then, the repaired short fragments were ligated with illumina paired-end adapters to the ends. After the library was constructed, the Agilent 2100 and library Qubit 2.0 were used to check concentration and quality of the cDNA library while insert size were detected using (quantititive) Q-PCR method for the effective concentration of the library.

\section{Sequencing data analysis and assembly}

The raw data was first filtered to obtain high quality de novo transcriptome sequence data. Adapter sequences and low-quality sequences (reads with ambiguous bases 'N') were all removed from the raw data. The qualified reads were extended into contigs with trinity software through the overlap between sequences. Then the contigs were connected into transcript sequences, according to pair-end information of the sequences, which recovers full-length transcripts across a broad range of expression levels, with sensitivity similar to other methods that rely on genome alignment [10]. The overall settings used for this assembly was using de Bruijn graph algorithm by kmer with other parameters set at default levels for Trinity [10].

\section{Gene annotation}

Using BLAST software [49] with an E-value parameter not greater than $10^{-5}$ and HMMER parameter E-value not more than $10^{-10}$, assembled sequences were compared against the National Center for Biotechnology Information (NCBI) non-redundant (Nr) [50] (http://www.ncbi.nlm.nih.gov/), Swiss-Prot [51] GO [52], COG [53], KOG [54], and KEGG [55]. The gene ontology (GO) database annotates genes as belonging to one of the three functional categories, molecular function, biological process, or cellular component. The functional categories of these unigenes were identified using the GO database. The unigenes were also aligned to the NCBI clusters of orthologous groups (COG) database to predict and classify possible functions. Pathway assignments were determined with Kyoto Encyclopedia of Genes and Genome (KEGG) pathway database using blast with E-value threshold of $10 \mathrm{E}^{-5}$. After predicting the unigene sequences of amino acids using HMMER software, Pfam database was used to identify the structure of protein domain sequences, and the prediction of protein functions [56]. Fiber biosynthesis and secondary metabolite biosynthesis genes pathway were identified according to the KEGG pathway database.

\section{Real-Time PCR verification}

Four genes potentially involved in the biosynthesis of cellulose, a component of fiber, were selected for RealTime PCR experiments. Gene-specific quantitative realtime PCR primers were designed using the Primer Premier v5.0 software (Premier Biosoft, USA). The details regarding the genes and primers are presented in Additional file 5. Total RNA was extracted from the young leaves, young seeds, flower buds, and fiber tissues using the RnaEx ${ }^{\text {Tix }}$ (GENEray Shanghai, China) as described above. One microgram RNA was used in reverse transcription with PrimeScript ${ }^{\mathrm{ma}}$ RT reagent kit (Perfect Real-Time) with gDNA eraser (Takara, China) according to manufacturer's guidelines. All primer pairs were examined using standard real-time PCR and Premix Ex Taq $(\mathrm{TaKaRa})$, and the presence of a single amplification product of the expected size for each gene was verified by electrophoresis on a $1.0 \%$ agarose gel with ethidium bromide staining. Real-time PCR was performed using a SYBR Premix ExTaq Kit (TaKaRa) on CFX96 $^{\text {ti }}$ RealTime Detection System (Bio-Rad, USA). The total volume of the reaction mixture was $25 \mu \mathrm{L}$ which contained $2 \mu \mathrm{L}$ cDNA, Template $(<100 \mathrm{ng}) 12.5 \mu \mathrm{L}$ SYBR Premix Ex Taq, (Tli RNaseH Plus) (2X), $0.5 \mu \mathrm{L}$ of Forward Primer and reverse primer each at a concentration of 10 pmol and $9.5 \mu \mathrm{L}$ of $\mathrm{dH} 2 \mathrm{O}$ (sterile distilled water).

PCR conditions were as follows: $95{ }^{\circ} \mathrm{C}$ for $30 \mathrm{~s}$ initial denaturation, followed by 40 cycles of denaturation at $95{ }^{\circ} \mathrm{C}$ for five seconds and annealing at $60{ }^{\circ} \mathrm{C}$ for $30 \mathrm{~s}$. All reactions were carried out in triplicate for technical 
and biological repetitions and the amplicons were subject to melting curve analysis to determine amplification specificity. Raw data on the relative abundance of each transcript were expressed as mean \pm standard deviation (SD). The relative expression levels of selected unigenes were normalized to actin gene (unigene c26600) and calculated using the 2- $\Delta \Delta \mathrm{Ct}$ method [57]. A melting curve was generated for each sample at the end of each run to assess the purity of the amplified products.

\section{SSR mining}

The identification and discovery of microsatellites loci was accomplished by use of the microsatellite identification tool, MIcroSAtellite (MISA), which is able to completely identify microsatellites loci (http://pgrc.ipk-gatersleben.de/misa/). The search criteria were set for identification of perfect mono-, di-, tri-, tetra-, penta-, and hexa-nucleotide motifs with a minimum of ten, six, five, four, four, and four repeats, respectively. Mononucleotide repeats were ignored during subsequent analysis. Subsequently, primer pairs were designed for genes with SSRs using the Primer3 (version 2.23) with default settings $[47,58]$, and the PCR product size ranging from 100 to $280 \mathrm{bp}$ (Additional file 4).

\section{Availability of supporting data}

All the sequence raw data generated from this study have been submitted to the NCBI (National Center for Biotechnology Information) Short Read Archive under accession number SRX1020451. http://www.ncbi.nlm.nih.gov/sra.

\section{Additional files}

\section{Additional file 1: KEGG pathways of the assembled unigenes. (PDF $69 \mathrm{~kb}$ )}

Additional file 2: Transcripts involved in fiber biosynthesis. (XLS $22 \mathrm{~kb}$ )

Additional file 3: Annotated transcriptional factors involved in stress response. (XLS $44 \mathrm{~kb}$ )

Additional file 4: Simple Sequence Repeats (SSR) loci generated from the transcriptome. A detailed information on primers for the 5,693 SSRs and the frequency of SSR motifs in Sodom apple unigenes, and are respectively presented on sheet 1 and 2 of this file. (XLS $8878 \mathrm{~kb}$ )

Additional file 5: Primers for Real-Time PCR analysis. Primers were designed using primer premier program (version 5.0). (XLS $20 \mathrm{~kb}$ )

\section{Abbreviations}

ESTs: Expressed Sequence Tags; SSRs: Simple sequence repeats; NCBI: National Center for Biotechnology Information; BLAST: Basic Local Alignment Search Tool; KEGG: Kyoto Encyclopedia of Genes and Genomes; COG: Clusters of Orthologous Groups; KOG: Eukaryotic Orthologous Groups of proteins; Pfam: Protein families; GO: Gene ontology;

PGM: Phosphoglucomutase; UGP2: Glucose-1- phosphate uridyldyltransferase; GAE: UDP-D-glucuronate-4-epimerase; GAUT: alpha-1,4-

galacturonosyltransferase; C4H: Cinnate 4-hydroxylase.

\section{Competing interests}

The authors declare they have no competing interests.

\section{Authors' contributions}

NM and WX carried out sequence data analysis and drafted the manuscript NM performed the experiments. AM and JX contributed to sample collection and manuscript revision. AL designed and managed the experiments, and organized the manuscript. All authors read and approved the final version of the manuscript.

\section{Acknowledgments}

This study was funded through the support of the Chinese Hunan Yunjin Group Co. Ltd. We would also like to thank the CGIAR Research Program 6: Forest, Trees and Agroforestry, for partially funding this work. We are also grateful to Mr. AO Tao and Ms. Zhao Jaiwen for assisting with sample collection.

\section{Author details}

'Key Laboratory for Economic Plants and Biotechnology, Kunming Institute of Botany, Chinese Academy of Sciences, Lanhei Road 132, Heilongtan, Kunming 650201, Yunnan, China. ${ }^{2}$ University of Chinese Academy of Sciences, Beijing 100049, China. ${ }^{3}$ World Agroforestry Centre, East and Central Asia Office, 132 Lanhei Road, Kunming 650201, China. ${ }^{4}$ World Agroforestry Centre (ICRAF), P.O. Box 30677-00100, Nairobi, Kenya.

Received: 17 June 2015 Accepted: 9 September 2015

Published online: 22 September 2015

\section{References}

1. Tao P, Gilbert MG, Stevens WD. Flora of China. 1995;16:189-270 www.eflora.org. Accessed 3 March 2015.

2. Chen $\mathrm{Q}$, Zhao T, Wang M, Wang J. Studies of the fibre structure and dyeing properties of Calotropis gigantea, kapok and cotton fibres. Color Technol. 2013. doi:10.1111/cote.1205.

3. Lebrun J. Catalogue des plantes vasculaires de la Mauritanie et du Sahara occidental, vol. 55. Genève: Boissiera; 1998.

4. Orwa C, Mutua A, Kindt R, Jamnadass R, Simons A. Agroforestree database: a tree reference and selection guide version 4.0. 2009; http://www .worldagroforestry.org/af/treedb/. Accessed 17 March 2015

5. Cheema HN, Bashir A, Khatoon A, labal N, Zafar Y, Malik KA. Molecular characterization and transcriptome profiling of expansin genes isolated from Calotropis procera fibers. Electron J Biotechnol. 2010;13:10-1.

6. Mueen A, Rana A, Dixit V. Calotropis species (Asclepediaceae), a comprehensive review. Pharmacogn Mag. 2005;1:48-52.

7. Aderounmu AO, Omonisi AE, Akingbasote JA, Makanjuola M, Bejide RA, Orafidiya LO, et al. Wound-healing and potential anti-keloidal properties of the latex of Calotropis procera (Aiton) Asclepiadaceae in rabbits. Afr J Tradit complement Altern Med. 2013;10:574-9.

8. Kumar PS, Suresh E, Kalavathy S. Review on a potential herb Calotropis gigantea (L.). R Br Sch Acad Pharm. 2013;2:135-43.

9. Wang Z, Gerstein M, Snyder M. RNA-SEQ: A revolutionary tool for transcriptomics. Nat Rev Genet. 2009:170:1945-56.

10. Grabherr MG, Haas BJ, Yassour M, Levin JZ, Thompson DA, Amit I, et al. A Full length transcriptome assembly from RNA Seq data without a reference genome. Nat Biotechnol. 2011;29(7):644-52.

11. Galla G, Barcaccia G, Ramina A, Collani S, Alagna F, Baldoni L. Computational annotation of genes differentially expressed along olive fruit development. BMC Plant Biol. 2009;9:128.

12. Kang C, Darwish O, Geretz A, Shahan R, Alkharouf N, Liu Z. Genome-scale transcriptomic insights into early-stage fruit development in woodland strawberry Fragaria vesca. Plant Cell. 2013;25:1960-78.

13. Sangwan RS, Tripathi S, Singh J, Lokesh K, Narnoliya LK, Neelam S, et al. De novo sequencing and assembly of Centella asiatica leaf transcriptome for mapping of structural, functional and regulatory genes with special reference to secondary metabolism. Gene. 2013;525:58-76.

14. Liu T, Zhu S, Tang Q, Chen P, Yu Y, Tang S. De novo assembly and characterization of transcriptome using Illumina paired-end sequencing and identification of CesA gene in ramie (Boehmeria nivea L. Gaud). BMC Genomics. 2013;14:125.

15. Yang $Y, X u M, L u o ~ Q$, Wang J, Li H. De novo transcriptome analysis of Liriodendron chinense petals and leaves by illumina sequencing. Gene. 2014;534:155-62 
16. Xu P, Liu Z, Fan X, Gao J, Zhang X, Shen X. De novo transcriptome sequencing and comparative analysis of differentially expressed genes in Gossypium aridum under salt stress. Gene. 2013;525:26-34.

17. Yoo MJ, Wendel JF. Comparative evolutionary and developmental dynamics of the cotton (Gossypium hirsutum) fiber transcriptome. PLoS Genet. 2014;10, e1004073. doi:10.1371/journal. pgen.1004073.

18. Lander ES, Linton LM, Birren B, Nusbaum C, Zody MC, Baldwin J, et al. Initial sequencing and analysis of the human genome. Nature. 2001;doi:10.1038/ 35057062

19. Ge X, Chen H, Wang H, Shi A, Liu K. De novo assembly and annotation of Salvia splendens transcriptome using the illumina platform. PLoS One. 2014;9(3), e87693. doi:10.1371/journal.pone.0087693.

20. Fu N, Wang Q, Shen HL. De novo assembly, gene annotation and marker development using illumina paired-end transcriptome sequences in celery(Apium graveolens L.) 2013;doi:10.1371/journal.pone.0057686.

21. Wang X, Xu R, Wang R, Liu A. Transcriptome analysis of Sacha Inchi (Plukenetia volubilis L.) seeds at two developmental stages. BMC Genomics. 2012;13:716.

22. Upadhyay S, Phukan U, Mishra S, Kumar R. De novo leaf and root transcriptome analysis identified novel genes involved in steroidal sapogenin biosynthesis in Asparagus racemosus. BMC Genomics. 2014;15:746

23. Jia XL, Wang GL, Xiong F, Yu XR, Xu ZS, Wang F, et al. De novo assembly, transcriptome characterization, lignin accumulation and anatomic characteristics: novel insights into lignin biosynthesis during celery leaf development. Scientific reports. 2015; doi:10.1038/srep08259.

24. Yang YW, Bian SM, Yao Y, Liu JY. Comparative proteomic analysis provides new insights into the fiber elongating process in cotton. J Proteome Res. 2008;7:4623-37.

25. Kumar M, Thammannagowda S, Bulone V, Chiang V, Han K, Joshi C, et al. An update on the nomenclature for the cellulose synthase genes in Populus. Trends Plant Sci. 2009;14:1360-85.

26. Endler A, Persson S. Cellulose synthases and synthesis in Arabidopsis. Mol Plant. 2011:4:199-211.

27. Richmond T, Somerville C. The cellulose synthase superfamily. Plant Physiol. 2000;124:495-8

28. Tanaka K, Murata K, Yamazaki M, Onosato K, Miyao A, Hirochika H. Three distinct rice cellulose synthase catalytic subunit genes required for cellulose synthesis in the secondary wall. Plant Physiol. 2003;133:73-83.

29. Kim H, Triplett B, Zhang H, Lee M, Hinchliffe D, Li P, et al. Cloning and characterization of homeologous cellulose synthase catalytic subunit 2 genes from allotetraploid cotton (Gossypium hirsutum L.). Gene. 2012:494:181-9.

30. Kazumasa A, Takeshi I, Tatsuya T, Toshio A, Sumihiro H. Successive gycosyltransferase activity and enzymatic characterization of pectic poly gacturonate 4-galacturonosyltransferase solubilized from pollen tubes of petunia axillaris using pyridylaminated oligogalacturonates as substrates. Plant Physiol. 2002;130:374-9.

31. Scheller HV, Doong RL, Ridley BL, Mohnen D. Pectin biosynthesis: a solubilized galacturonosyltransferase from tobacco catalyzes the transfer of galacturonic acid from UDP-galacturonic acid on to the non-reducing end of homogalacturonan. Planta. 1999;207:512-7.

32. Liljebjeike K, Adolphoson R, Baker K, Doong RL, Mohnera D. Enzymatics synthesis and purification of uridine diphosphate $\left[{ }^{14} \mathrm{C}\right]$ galacturonic acid; a substrate for pectin biosynthesis. Res. 1995;187:261-72.

33. Sterling JD, Atmodjo MA, Inwood SE, Kumar Kolli VS, Quigley HF, Hahn MG. Functional identification of an Arabidopsis pectin biosynthetic homogalacturonan galacturonosyltransferase. Proc Natl Acad Sci. 2006;103:5236-41. USA.

34. Wang H, Guo Y, LV F, Zhu H, Wu S, Jiang Y, et al. The essential role of GhPEL gene, encoding a pectate lyase, in cell wall loosening by depolymerization of the de-esterified pectin during fiber elongation in cotton. Plant Mol Biol. 2010;72:397-406.

35. Boerjan W, Ralph J, Baucher M. Lignin Biosynthesis. Annu Rev Plant Biol. 2003;54:519-46. ISSN 1040-2519.

36. Ambavaram R, Krishnan A, Trijatmiko R, Pereira A. Coordinated activation of cellulose and repression of lignin biosynthesis pathways in rice. Plant Physiol. 2011;155:916-31.

37. Singh B, Rastogi RP. Structure of Asclepin and some observations on the NMR spectra of Calotropis glycosides. Phytochemistry. 1972;11:757-62.
38. Kitagawa I, Zhang RS, Park JD, Baek NI, Takeda Y, Yoshikawa M, et al. Indonesian medicinal plants: Chemical structures of Calotroposides A and B, two new oxypregnane-oligoglycosides $A$ and $B$, from the root of Calotropis gigantea (Asclepiadaceae). Chem Pharm Bull. 1992:40:2007-13.

39. Sumangala KB, Sharma A. Therapeutic potential of cardiac glycoside of Calotropis gigantea for breast cancer. Int Res J Pharm. 2013;4:6.

40. Eisenreich W, Rohdich F, Bacher A. Deoxyxylulose phosphate pathway to terpenoids. Trends Plant Sci. 2001;6:78-84.

41. Facchini PJ. Alkaloid biosynthesis in plants: biochemistry, cell biology, molecular regulation, and metabolic engineering applications. Annu Rev Plant Biol. 2001;52:29-66.

42. Burbulis IE, Shirley BW. Interactions among enzymes of the Arabidopsis flavonoid biosynthetic pathway. Proc Natl Acad Sci. 1999;96:12929-34.

43. Anterola AM, Jeon JH, Davin LB, Lewis NG. Transcriptional control of monolignol biosynthesis in Pinus taeda factors affecting monolignol ratios and carbon allocation in phenylpropanoid metabolism. J Biol Chem. 2002;277:18272-80.

44. Li GX, Wu MS, He CY. Resistance to high salt and cold stress of transgenic rice seedlings with over-expressed and RNAi-silenced OsBTF3. Chin J Rice Sci. 2012;26:5-8

45. Jakoby M, Weisahaar B, Droge-Laser W, Vicente-Carbajosa J, Tiedemann J, Kroj T, et al. bZIP trancription factor in Arabidopsis. Trends Plant Sci. 2002;106-11.

46. Abe H, Yamaguchi-Shinozaki K, Urao T, Iwasaki T, Hosokawa D, Shinozaki K Role of arabidopsis MYC and MYB homologs in drought and abscisic acidregulated gene expression. Plant Cell. 1997;9:1859-68.

47. Varshney RK, Sigmund R, Borner A, Korzun V, Stein NR, Sorrells ME. Interspecific transferability and comparative mapping of barley EST-SSR markers in wheat, rye and rice. Plant Sci. 2005;168:195-202. doi:10.1016/j.

48. Liu Z, Chen T, Ma L, Zhao Z, Zhao PX, Nan Z, et al. Global Transcriptome Sequencing Using the Illumina Platform and the Development of EST SSR Markers in Autotetraploid Alfalfa. PLoS One. 2011;8(12):e83549.

49. Altschul SF, Madden TL, Schäffer AA, Zhang J, Zhang Z, Miller W, et al. Gapped BLAST and PSI BLAST: A New Generation of Protein Database Search Programs. Nucleic Acids Res. 1997;25:3389-402.

50. Deng YY, Li JQ, Wu SF, Zhu Y, Chen Y, He F. Integrated nr Database in Protein Annotation System and Its Localization. Comput Eng. 2006:32:71-4.

51. Apweiler R, Bairoch A, Wu CH, Barker WC, Boeckmann B, Ferro S. UniProt: the Universal Protein knowledge base. Nucleic Acids Res. 2004;32(Database issue):D115-9.

52. Ashburner M, Ball CA, Blake JA, Botstein D, Butler HJ, Cherry M, et al. Gene ontology:tool for the unification of biology. Nat Genet. 2000;25:25-9.

53. Tatusov RL, Galperin MY, Natale DA. The COG database: a tool for genome scale analysis of protein functions and evolution. Nucleic Acids Res. 2000;28:33-6.

54. Koonin EV, Fedorova ND, Jackson JD, Jacobs AR, Krylov DM, Makarova KS, et al. A comprehensive evolutionary classification of proteins encoded in complete eukaryotic genomes. Genome Biol. 2004;5:R7.

55. Kanehisa M, Goto S, Kawashima S, Okuno Y, Hattori M. The KEGG resource for deciphering the genome. Nucleic Acids Res. 2004;32:D277-80.

56. Finn RD, Bateman A, Clements J, Coggill P, Eberhardt RY, Eddy SR, et al. Pfam: the protein families database. Nucleic Acids Res. 2013;42:D222-30 doi:10.1093/nar/gkt1223.

57. Livak KJ, Schmittgen TD. Analysis of relative gene expression data using 0-time quantitative PCR and the 2-DDCT method. Methods. 2001;25:402-8.

58. Rozen S, Skaletsky H. Primer3 on the WWW for general users and for biologist programmers. Methods Mol Biol. 2000;132:365-86. 